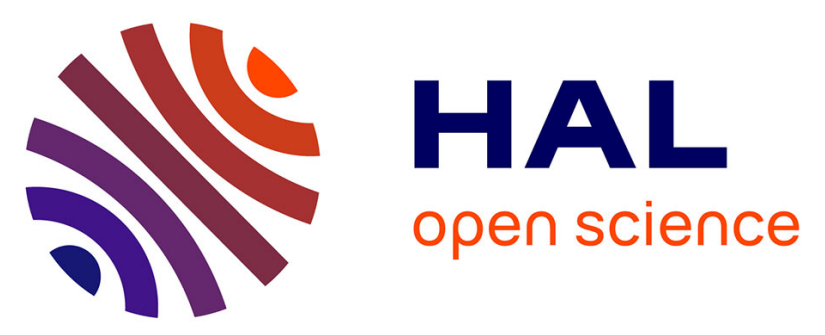

\title{
Élaboration des valeurs biologiques françaises en vue de la mise à disposition de valeurs biologiques d'interprétation pour la surveillance biologique des expositions professionnelles
}

Fatoumata Sissoko, Dominique Brunet, Marie-Laure Cointot, Florence

Pillière, Anne Maitre, Irène Sari-Minodier, Claude Viau

\section{To cite this version:}

Fatoumata Sissoko, Dominique Brunet, Marie-Laure Cointot, Florence Pillière, Anne Maitre, et al.. Élaboration des valeurs biologiques françaises en vue de la mise à disposition de valeurs biologiques d'interprétation pour la surveillance biologique des expositions professionnelles. Toxicologie Analytique et Clinique, 2017, 29 (4), pp.377 - 386. 10.1016/j.toxac.2017.06.004 . hal-01794438

\section{HAL Id: hal-01794438 \\ https://hal-amu.archives-ouvertes.fr/hal-01794438}

Submitted on 17 May 2018

HAL is a multi-disciplinary open access archive for the deposit and dissemination of scientific research documents, whether they are published or not. The documents may come from teaching and research institutions in France or abroad, or from public or private research centers.
L'archive ouverte pluridisciplinaire $\mathbf{H A L}$, est destinée au dépôt et à la diffusion de documents scientifiques de niveau recherche, publiés ou non, émanant des établissements d'enseignement et de recherche français ou étrangers, des laboratoires publics ou privés. 


\title{
Élaboration des valeurs biologiques françaises en vue de la mise à disposition de valeurs biologiques d'interprétation pour la surveillance biologique des expositions professionnelles
}

Derivation of French biological values for the provision of biological interpretation values for the biomonitoring of occupational exposures

\author{
Fatoumata Sissoko ${ }^{a, *}$, Dominique Brunet $^{a}$, \\ Marie-Laure Cointot ${ }^{a}$, Florence Pillière ${ }^{b}$, \\ Anne Maître $^{c}$, Irène Sari-Minodier ${ }^{d}$, Claude Viau ${ }^{e}$
a Direction de l'évaluation des risques, unité d'évaluation des substances chimiques, 14, rue Pierre-et-Marie-Curie, Anses, 94701 Maisons-Alfort cedex, France
${ }^{\mathrm{b}}$ Département études et assistance médicales, INRS, 65, boulevard Richard-Lenoir, 75011
Paris, France
' Laboratoire de toxicologie professionnelle et environnementale, équipe EPSP, laboratoire TIMC (UMR CNRS 5525), DBTP, IBP, CHU de Grenoble-Alpes, France
¿ Service de médecine et santé au travail/IMBE (UMR 7263), faculté de médecine, université de Aix-Marseille, 27, boulevard Jean-Moulin, 13385 Marseille cedex 05, France
e Département de santé environnementale et santé au travail, institut de recherche en santé publique, université de Montréal, Montréal, Canada

MOTS CLÉS

Surveillance

biologique ;
Résumé Pour être interprétés, les résultats de la surveillance biologique des expositions professionnelles aux agents chimiques doivent pouvoir être comparés à des valeurs biologiques d'interprétation. Parmi ces valeurs figurent les valeurs élaborées en France par l'Anses selon une

* Auteur correspondant.

Adresse e-mail : fatoumata.sissoko@anses.fr (F. Sissoko). 


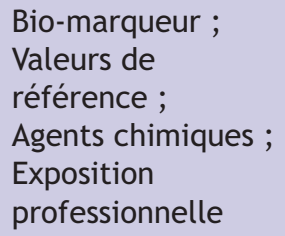

\section{KEYWORDS}

Biological monitoring; Biomonitoring;

Biomarker;

Reference values;

Chemicals;

Occupational

exposure démarche structurée s'appuyant sur des collectifs d'experts mettant en œuvre une expertise scientifique indépendante, collective et pluridisciplinaire. Deux types de valeurs biologiques en milieu professionnel sont recommandés par l'Anses : des valeurs limites biologiques et des valeurs biologiques de référence. Ces valeurs sont élaborées sur la base des données disponibles dans la littérature scientifique. L'élaboration par l'Anses des valeurs pouvant être utilisées dans le cadre d'une surveillance biologique des expositions professionnelles dépend de la nature du polluant étudié. Selon que la substance est un toxique à seuil d'effet ou sans seuil d'effet, tel qu'un cancérogène génotoxique, l'approche sera différente. Après présentation de cette méthodologie, celle-ci est illustrée au travers de quelques exemples (cadmium, styrène, 2butoxyéthanol et acrylamide) afin que leur utilisation par les médecins du travail comme valeurs biologiques d'interprétation puisse être menée de façon éclairée.

๑ 2017 Société Française de Toxicologie Analytique. Publié par Elsevier Masson SAS. Tous droits réservés.

Summary Adequate interpretation of biomonitoring results of occupational exposures to chemicals requires comparison values also referred to as biological interpretation values. Among these values are those derived in France by Anses, which are based on a structured approach led collectively by independent and multidisciplinary groups of scientific experts. Two types of occupational biological values are recommended by Anses: biological limit values and biological reference values. These values are derived from the data available in the scientific literature. The article first shows that the derivation by Anses of the biological values to be used for biomonitoring of occupational exposures is linked to the nature of the chemical examined. Depending upon whether the substance is a threshold or non-threshold toxicant, such as genotoxic carcinogens, the approach will be different. Next, some specific examples are presented (cadmium, styrene, 2-butoxyethanol and acrylamide) to help occupational physicians with the informed interpretation of biomonitoring results collected in workplaces.

(c) 2017 Société Française de Toxicologie Analytique. Published by Elsevier Masson SAS. All rights reserved.

\section{Introduction}

La surveillance biologique des expositions professionnelles (SBEP) ou bio-métrologie a été définie dès les années 1980 par la Commission des communautés européennes (CEC), le National Institute of Occupational Safety and Health (NIOSH) et l'Occupational Safety and Health Admnistration (OSHA) comme «l'identification et la mesure des substances de l'environnement du poste de travail ou de leurs métabolites dans les tissus, les excrétas, les sécrétions ou l'air expiré des salariés exposés, pour évaluer l'exposition et les risques pour la santé, en comparant les valeurs mesurées à des références appropriées ». Les paramètres mesurés sont appelés indicateurs biologiques d'exposition (IBE) ou biomarqueurs d'exposition [1]. Un IBE peut être défini comme étant la substance mère, ou un de ses métabolites, mesurés dans un milieu biologique dont la variation est associée à une exposition à l'agent visé par I'IBE. Des indicateurs biologiques d'effets précoces et réversibles s'ajoutent à cette définition dans la mesure où ils peuvent être spécifiquement corrélés à l'exposition professionnelle [2]. Cependant, l'interprétation des résultats de la SBEP nécessite que ces derniers soient comparés à des valeurs de référence appropriées, dénommées au niveau français valeurs biologiques d'interprétation (VBI) [3]. II existe plusieurs organismes, français ou internationaux, qui élaborent des valeurs biologiques pouvant être utilisées comme VBI pour la SBEP $[4,5]$. Certains organismes précisent comment ils élaborent leurs valeurs biologiques [6-10].

Il s'agit de décrire dans cet article la méthodologie d'élaboration des différentes valeurs recommandées par l'Anses en vue d'une utilisation dans le cadre d'une SBEP et de l'illustrer à travers quelques exemples (cadmium, 2-butoxyethanol, styrène et acrylamide) afin que leur utilisation par les médecins du travail comme VBI puisse être menée de façon éclairée.

\section{Définition des valeurs élaborées en France par l'Anses pour la surveillance biologique des expositions professionnelles aux agents chimiques}

Selon les substances et le corpus de données, deux types de valeurs biologiques en milieu professionnel sont recommandés par l'Anses : des valeurs limites biologiques (VLB) et des valeurs biologiques de référence (VBR). Ces valeurs 
sont élaborées sur la base des données disponibles dans la littérature scientifique.

Les VLB pour des sujets professionnellement exposés correspondent aux valeurs limitées des biomarqueurs ${ }^{1}$ jugés pertinents. Elles visent à prévenir la survenue d'effets sanitaires chez les personnes professionnellement exposées aux substances évaluées. Plusieurs types de VLB peuvent être recommandés :

- une VLB basée sur un effet sanitaire : elle correspond à la concentration de l'IBE à laquelle les données scientifiques ne rapportent pas d'effets sanitaires pour les substances à seuil ; en ce qui concerne les substances cancérogènes sans seuil, les concentrations de l'IBE correspondant à des niveaux de risque donnés sont estimées. Dans tous les cas, il faut que le corpus de données scientifiques soit suffisant pour déterminer avec certitude une relation dose-réponse ;

- une VLB basée sur une exposition à la VLEP-8 $\mathrm{h}$ : il s'agit de la concentration moyenne de l'IBE correspondant à une exposition des travailleurs à la VLEP- $8 \mathrm{~h}$; cette concentration peut être, soit issue de publications qui permettent de définir des équations de droites de régression entre les concentrations de biomarqueurs et les concentrations atmosphériques, soit prédites par des modèles toxicocinétiques ;

- une VLB pragmatique, notamment pour les substances cancérogènes : en effet, en l'absence de données quantitatives suffisantes, une VLB pragmatique peut être proposée sur la base d'un effet sanitaire autre que le cancer. Cette valeur pragmatique ne garantit pas l'absence d'effet sanitaire mais vise à limiter les expositions à ces substances sur les lieux de travail.

Lorsque cela est possible, des VBR sont également proposées. Celles-ci correspondent à des niveaux d'imprégnation mesurés dans une population générale d'adultes dont les caractéristiques sont proches de celles de la population française ou dans une population de témoins non professionnellement exposés à la substance d'intérêt. Les VBR ne peuvent être considérées comme protectrices de l'apparition d'effets sanitaires. Néanmoins elles permettent de comparer les concentrations de biomarqueurs mesurées chez des professionnels exposés aux valeurs observées en l'absence d'exposition en milieu de travail. Ces valeurs sont particulièrement intéressantes dans les cas où il n'est pas possible d'élaborer une VLB [2].

\section{Principes d'élaboration des valeurs de l'Anses pour la surveillance biologique des expositions professionnelles}

L'élaboration des VLB et des VBR par les comités d'experts de l'Anses suit une approche structurée impliquant une expertise collective pluridisciplinaire et indépendante permettant de garantir au mieux la qualité scientifique. Cette expertise s'appuie notamment sur deux collectifs d'experts (le comité d'experts spécialisés « expertise en vue de

\footnotetext{
${ }^{1}$ Le terme biomarqueur fait référence à la fois aux IBE et aux indicateurs biologiques d'effet.
}

la fixation de valeurs limites à des agents chimiques en milieu professionnel » et le groupe de travail « indicateurs biologiques d'exposition »). Ces groupes pluridisciplinaires d'experts sont composés d'experts : toxicologues, médecins du travail, épidémiologistes, chimistes, modélisateurs, pharmaciens biologistes, hygiénistes du travail, évaluateurs de risques. Un guide méthodologique, auquel il est fait référence dans la suite de cet article, est publié sur le site de l'Anses [2]. Il présente le mode d'élaboration de ces valeurs et fait l'objet d'une actualisation tous les 3 ans, à l'occasion du renouvellement des comités d'experts.

La première étape consiste à recenser les données disponibles dans la littérature scientifique relatives aux éléments de toxicocinétique et toxicodynamique de la substance mère et des IBE retenus, à leur spécificité, de même qu'aux données pouvant affecter l'interprétation des résultats. Sont également recherchées les informations concernant les relations entre les concentrations des IBE et les effets sanitaires d'une part, les relations entre les concentrations des IBE et les expositions d'autre part (études de terrain et/ou chez des volontaires, études expérimentales, modèles pharmacocinétiques). Des études publiées permettent également d'identifier des niveaux d'IBE retrouvés dans la population générale et/ou chez des témoins non professionnellement exposés. Des données sur les conditions de prélèvement et la stabilité des échantillons biologiques sont également recensées. Les méthodes analytiques décrites dans la littérature pour le dosage des IBE retenus, sont également rapportées avec l'objectif de renseigner succinctement certains paramètres spécifiques aux méthodes analytiques et non de recommander une méthode pour le dosage. L'ensemble de ces informations sur les avantages et inconvénients (sensibilité, spécificité, faisabilité, corrélations...) sur chacun des IBE sont ainsi listés.

À partir de l'ensemble des données disponibles, les experts identifient tout d'abord parmi les IBE existants (voire parfois les indicateurs biologiques d'effets) ceux qu'ils jugent pertinents pour mettre en place une SBEP. Leur spécificité vis-à-vis de l'exposition à l'agent chimique d'intérêt, leur durée de présence dans l'organisme (la demi-vie déterminant le moment de prélèvement), la nature des prélèvements (caractère invasif ou non, selon le milieu biologique concerné) sont évalués ainsi que les méthodes de conservation des prélèvements. Des précisions sur l'ensemble des éléments à examiner permettant de déterminer ce qu'est un bon indicateur biologique d'exposition à un agent chimique ont été publiées récemment [3]. Cette évaluation permet de sélectionner les biomarqueurs jugés pertinents pour lesquels la construction de valeurs biologiques va être recherchée. Il peut ainsi exister un ou plusieurs IBE sans qu'il ne leur soit nécessairement associé de VLB ni de VBR.

\section{Méthode d'élaboration des VLB}

Le mode de construction des VLB dépend notamment de la nature des données disponibles et du mode d'action des substances.

La construction des VLB suit un arbre décisionnel guidé par le mode d'action de la substance, qui peut être soit à seuil d'effet soit sans seuil d'effet (Fig. 1). 


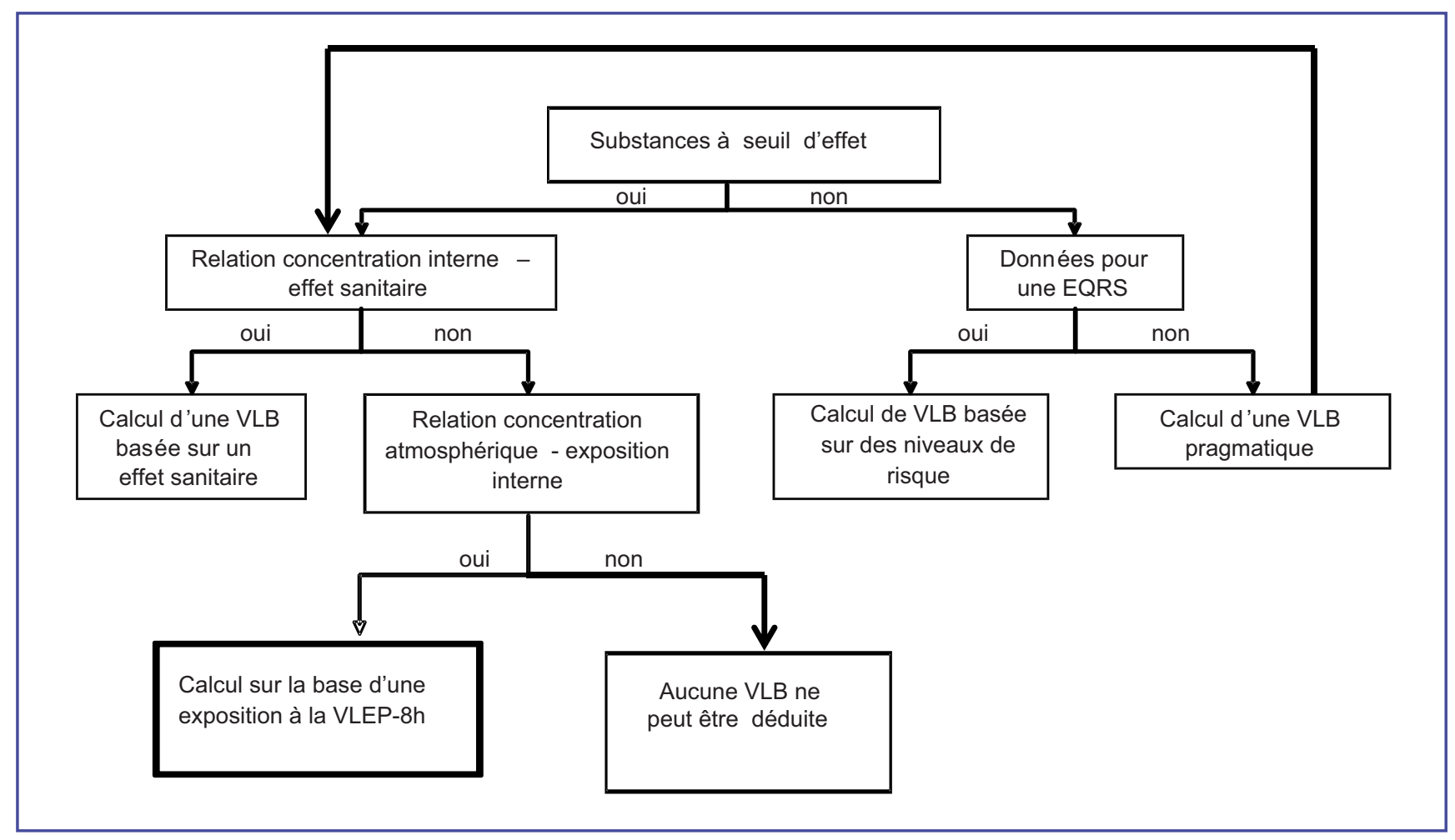

Figure 1. Arbre décisionnel pour l'élaboration d'une VLB (adapté de [2]). EQRS : évaluation quantitative de risque sanitaire ; VLB : valeur limite biologique ; VLEP : valeur limite d'exposition professionnelle.

Pour les substances dites à seuil d'effet, il est admis l'existence d'un seuil de toxicité en dessous duquel aucun effet néfaste sur la santé n'est attendu. L'effet toxique ne survient qu'au-delà d'une certaine dose qui dépasse les capacités de l'organisme à compenser ou à s'adapter [2]. Dans le cas des substances sans seuil d'effet, l'effet peut apparaître quelle que soit la dose reçue et la probabilité de survenue augmente le plus souvent avec la dose. Il s'agit, pour l'essentiel, des effets cancérogènes génotoxiques directs [11].

Pour les biomarqueurs jugés pertinents, il s'agit d'examiner en fonction du mode d'action de la substance, la nature des données disponibles permettant de mettre en relation les concentrations de ces biomarqueurs soit avec un effet sanitaire, soit avec une exposition atmosphérique à la VLEP-8 $\mathrm{h}$.

\section{Cas de l'élaboration d'une VLB pour une substance à seuil d'effet basée sur un effet sanitaire}

Pour les substances à seuil d'effet, les données humaines et expérimentales sont étudiées afin de rechercher une relation dose-réponse entre les concentrations d'IBE et l'apparition d'effets sanitaires. Lorsqu'il existe des relations doses/réponses entre les concentrations d'IBE et plusieurs effets sanitaires, il est alors nécessaire de choisir un effet dit « critique », parmi les effets adverses jugés pertinents. Il s'agira habituellement de l'effet survenant aux plus faibles concentrations d'exposition. Cette étape implique d'identifier une concentration maximale pour laquelle l'effet critique n'est pas observé (No-Observed-Adverse
Effect Concentration [NOAEC]) et/ou une concentration minimale pour laquelle l'effet critique est observé (LowestObserved-Adverse-Effect Concentration [LOAEC]). Lorsque les données s'y prêtent, l'utilisation de l'approche Benchmark dose (BMD) est privilégiée par rapport à l'approche traditionnelle (identification du couple NOAEC/LOAEC). L'approche BMD permet d'estimer la dose correspondant à un niveau de réponse défini ou à un pourcentage défini de réponse supplémentaire par rapport aux témoins. La dose critique utilisée est alors la BMDL (la limite inférieure de l'intervalle de confiance de la BMD). La concentration retenue (BMDL, NOAEC ou LOAEC) est ensuite pondérée par des facteurs d'ajustement.

Les choix de l'effet critique à retenir, des concentrations de biomarqueurs, des données toxicocinétiques et des facteurs d'ajustement à appliquer en fonction des études disponibles pour élaborer une VLB sont soumis à expertise collective et argumentés. Il est à noter que la démarche mise en œuvre est similaire à celle utilisée pour la construction des valeurs limites atmosphériques également élaborées à l'Anses.

\section{Cas de l'élaboration d'une VLB basée sur une exposition à la VLEP-8 $\mathrm{h}$}

Il est rarement possible de construire une VLB basée sur un effet sanitaire à partir des données humaines et peu d'études réalisées chez l'animal associent la survenue d'effets sanitaires à des concentrations de biomarqueurs.

L'approche alternative consiste alors à calculer la concentration moyenne de l'IBE correspondant à une exposition à la VLEP-8 $\mathrm{h}$. 
La relation entre la concentration de l'IBE et la concentration atmosphérique de la substance étudiée peut être établie soit à partir de la droite de régression entre l'IBE et les concentrations atmosphériques soit en utilisant des données de toxicocinétique (utilisation de modèles/équations de conservation de masse).

Utilisation de la corrélation entre une concentration atmosphérique et la concentration de l'IBE

Lorsqu'une corrélation peut être retrouvée entre les concentrations de l'IBE retenu et les concentrations atmosphériques de la substance étudiée, une VLB pourra être déduite de la VLEP-8 h à partir de l'équation de la droite de régression. La valeur sera donnée par la concentration moyenne correspondant à une exposition à la VLEP- $8 \mathrm{~h}$. Les VLB peuvent être déduites à partir d'études chez des volontaires et/ou d'études de terrain chez des travailleurs exposés. Il est à noter que des ajustements peuvent être nécessaires dans les études expérimentales chez des volontaires pour tenir compte des scénarios d'exposition en milieu professionnel, concernant notamment l'importance de la ventilation pulmonaire qui diffère habituellement entre ces deux groupes. Dans ce cas, il faut veiller à limiter les extrapolations afin de limiter les incertitudes assorties aux valeurs biologiques éventuellement recommandées.

Utilisation de la relation entre une exposition à la $V L E P-8 h$ et la concentration d'un IBE à partir de données toxicocinétiques (modélisation/équations de conservation de masse)

Il est possible, dans certain cas, de déterminer la relation entre la concentration de l'IBE et le niveau d'exposition (concentrations atmosphériques) à partir des données de toxicocinétique (modèles compartimentaux, modèles physiologically-based pharmacokinetic [PBPK], équations de conservation de masse). Il est alors possible de calculer la concentration d'IBE correspondant à la VLEP-8 h. L'utilisation de ce type d'approche indirecte introduit de nombreuses incertitudes dans la construction des VLB. Il est donc nécessaire de veiller à limiter le nombre d'extrapolations et à n'utiliser que les données de cinétique correspondant à des scénarios compatibles avec une exposition professionnelle (voie et durée d'exposition) ${ }^{2}$. Les modèles PBPK ne sont utilisés pour construire une VLB que si :

- ils ont été publiés dans la littérature scientifique et donc soumis à un comité de relecture et ;

- ils sont validés (avec des données indépendantes) pour l'inhalation et pour des scénarios d'exposition compatibles avec une exposition professionnelle pour l'indicateur biologique d'exposition considéré (pas d'extrapolation des concentrations d'un IBE à partir d'un autre $\mathrm{IBE}$ ).

Les choix des équations permettant de corréler les données d'exposition aux niveaux biologiques ou à des données de toxicocinétique (paramètres introduits dans le modèle modélisation) sont soumis à une expertise collective et argumentés.

\footnotetext{
2 Passage de l'animal à l'homme, de l'ingestion à l'inhalation, d'une exposition aiguë à chronique (pour la dose critique).
}

\section{Cas de l'élaboration d'une VLB pour une} substance cancérogène

\section{VLB basée sur un niveau de risque}

Dans le cas des substances considérées comme des cancérogènes sans seuil d'effet, lorsque l'information scientifique disponible permet de faire une évaluation quantitative de risque, la méthodologie prévoit d'exprimer les VLB sous forme d'une échelle de 3 concentrations de biomarqueurs correspondant aux trois excès de risque individuel $\left.(E R)^{3}\right)$ suivants : $10^{-4}, 10^{-5}$ et $10^{-6}$ (VLB basées sur des niveaux de risque).

\section{VLB pragmatique}

Pour les substances cancérogènes, en l'absence de données quantitatives suffisantes, la méthodologie prévoit d'élaborer une VLB pragmatique. Cette dernière approche consiste à déterminer les concentrations de biomarqueurs permettant de prévenir d'éventuels autres effets sanitaires en suivant la même démarche que celle décrite précédemment pour une VLB basée sur un effet sanitaire ou d'une VLB basée sur une exposition à une VLEP-8 $\mathrm{h}$. Il est important alors de garder à l'esprit que ces valeurs n'ont pas pour objectif de déterminer une valeur en dessous de laquelle il n'y a pas d'effet sanitaire cancérogène mais visent uniquement à mettre à disposition un outil afin de limiter les expositions à ces substances sur les lieux de travail. Dans certains cas, en l'absence d'études adéquates permettant de construire une VLB, seules des VBR peuvent être proposées.

\section{Propositions de VBR}

Les VBR sont définies à partir de valeurs retrouvées dans une population générale d'adultes dont les caractéristiques sont proches de celles de la population française ou dans une population de témoins non professionnellement exposés à la substance étudiée. Les VBR sont définies préférentiellement à partir de données en population générale pour les IBE afin de mettre en évidence une imprégnation hors de toute exposition professionnelle à l'agent chimique considéré. II faut donc pouvoir s'assurer de l'absence d'exposition, ce qui dans des études de terrain, ne peut pas toujours être certain et/ou vérifié même si la population témoin est considérée comme non exposée. Concernant les indicateurs biologiques d'effet, il est important de s'assurer que la population dans laquelle est mesurée le biomarqueur (population de référence) présente des caractéristiques proches de celle des travailleurs. En effet, il est nécessaire de prendre en compte certains paramètres comme l'état de santé de la population de référence (effet du travailleur sain). C'est pourquoi les VBR, pour les indicateurs biologiques d'effet, sont définis préférentiellement à partir de données de professionnels non exposés au polluant considéré.

Les valeurs retenues comme VBR correspondent le plus souvent à la valeur la plus proche du $95^{\mathrm{e}}$ pourcentile de la distribution des valeurs retrouvées au sein de la population considérée. Ces valeurs ne peuvent être considérées

\footnotetext{
${ }^{3}$ L'ERI exprime d'un point de vue théorique la probabilité supplémentaire d'observer l'effet néfaste, en particulier cancérogène, lié à la concentration d'exposition chez un individu.
} 
comme protectrices de l'apparition d'effets sanitaires mais elles permettent de comparer les concentrations de biomarqueurs mesurées chez des professionnels exposés aux valeurs observées en l'absence d'exposition en milieu de travail. Ces valeurs sont particulièrement intéressantes dans les cas où il n'est pas possible d'élaborer une VLB car elles permettent de mettre à disposition des médecins du travail a minima une valeur repère pour l'interprétation des résultats des dosages effectués dans le cadre d'une SBEP.

En ce qui concerne les données disponibles en population générale, les enquêtes nationales de bio-surveillance fournissant des données d'imprégnation en population générale de plusieurs pays sont utilisées, telles que l'Étude nationale nutrition santé (ENNS) française de Santé publique France, l'étude américaine du National Health and Nutrition Examination Survey (NHANES) ou l'étude allemande du German Environmental Survey (GerES). Dans la mesure du possible, ces enquêtes sont utilisées en donnant la priorité à l'étude française. En l'absence de donnée disponible à travers ces enquêtes nationales, des résultats publiés dans des articles sur des études en population générale à effectif plus restreint peuvent également être utilisés si elles sont jugées pertinentes.

\section{Illustration de la méthode d'élaboration des valeurs biologiques de l'Anses pour la surveillance biologique des expositions professionnelles}

\section{VLB pragmatique basée sur un effet sanitaire : cas du cadmium et de ses composés avec la proposition d'une VLB pour le cadmium urinaire [12]}

Le cadmium est considéré comme un cancérogène à seuil d'effet [13]. La littérature scientifique a permis d'identifier deux IBE d'intérêt pour la SBEP au cadmium et à ses composés, le cadmium sanguin et le cadmium urinaire. Deux indicateurs biologiques d'effets précoces ont également été identifiés : la béta-2-microglobuline $(\beta 2 M)$ et la protéine transporteuse de rétinol (RBP).

L'absence de données robustes ne permet pas de quantifier de relation entre les niveaux de ces IBE et les effets cancérogènes identifiés. Il n'a donc pas été possible de construire de VLB sur la base de la cancérogénicité du cadmium et de ses composés. Même si les données de la littérature ${ }^{4}$ permettent d'établir un lien potentiel de causalité entre les expositions au cadmium et une toxicité osseuse à long terme, là encore, aucune relation dose- réponse n'a pu être quantifiée (à partir de deux études de terrain disponibles).

La VLB pour le cadmium urinaire a donc été élaborée à partir des données de toxicité rénale du cadmium.

Il est accepté dans différentes études qu'une élévation de la concentration urinaire de la $\beta 2 M$ ou de la RBP au-dessus de $1000 \mu \mathrm{g} / \mathrm{g}$ de créatinine marque une

\footnotetext{
${ }^{4}$ Examinées jusqu'en 2012.
}

cytotoxicité tubulaire irréversible. Il est également admis qu'une concentration urinaire de RBP et/ou $\beta 2 \mathrm{M}$ supérieure à $300 \mu \mathrm{g} / \mathrm{g}$ de créatinine marque les premiers signes d'une cytotoxicité tubulaire [14-17]. C'est la raison pour laquelle une concentration urinaire de $300 \mu \mathrm{g} / \mathrm{g}$ de créatinine pour la RBP ou la $\beta 2 M$ est souvent retenue comme seuil de toxicité rénale du cadmium.

En ce qui concerne le cadmium urinaire, conformément à la méthodologie, les études récentes proposant des BMD sur des grands échantillons ont été examinées en détail [18-20]. Il n'a pas été jugé pertinent de retenir les résultats présentés dans le rapport de l'EFSA. En effet, la population visée est la population générale et les données étudiées proviennent d'une population très hétérogène qui ne comprend que $1 \%$ de travailleurs potentiellement exposés au cadmium.

La $\mathrm{BMD}_{10}{ }^{5}$ calculée par Jarup et Elinder correspondant à une concentration critique de $\beta 2 \mathrm{M}$ de $300 \mu \mathrm{g} / \mathrm{g}$ de créatinine est égale à $5 \mu \mathrm{g} / \mathrm{g}$ de créatinine pour les travailleurs de moins de 60 ans [20]. Il est à noter que l'intervalle de confiance à 90 ou $95 \%$ de cette valeur n'est pas renseigné dans la publication.

L'étude de Chaumont et al. est la seule rapportant une BMD (et l'intervalle de confiance) pour le cadmium urinaire sur une large population $(n=599)$ de travailleurs français, européens et américains en utilisant la réponse des marqueurs RBP et $\beta 2 M$ [18]. Cette étude présente certains avantages méthodologiques, comme l'effectif étudié qui exclut les fumeurs (ce qui permet ainsi de s'affranchir de l'influence du tabagisme pour la détermination de la valeur à recommander).

La concentration de $5,5 \mu \mathrm{g} / \mathrm{g}$ de créatinine de cadmium urinaire associée à une augmentation de la prévalence des concentrations urinaires de RBP ou de $\beta 2 M$ anormales ${ }^{6}$, arrondie à la valeur inférieure a été retenue. Une VLB pour le cadmium urinaire de $5 \mu \mathrm{g} / \mathrm{g}$ de créatinine a ainsi été proposée sur cette base.

L'âge moyen des travailleurs dans cette étude étant de 45 ans ( \pm 10 ans), il est possible que la VLB soit moins protectrice pour les jeunes travailleurs, étant donné l'accumulation potentielle du cadmium dans l'organisme au cours des années. Partant de ce postulat, les experts de l'Anses ont émis des recommandations de suivi médical complémentaire, initiant la mesure de marqueurs biologiques d'effets précoces de la cytotoxicité tubulaire. En effet, compte tenu du caractère cumulatif du cadmium dans l'organisme, il apparaît souhaitable de coupler le contrôle du respect de la VLB à un suivi complémentaire pour veiller à l'intégrité de la fonction rénale. Ce suivi devrait être déclenché à un niveau de concentration urinaire de cadmium, inférieur à la VLB. L'étude de Jarup et Elinder permet de déterminer une $\mathrm{BMD}_{10}$ pour le cadmium urinaire, à $1,5 \mu \mathrm{g} / \mathrm{g}$ de créatinine, associée à une élévation de la $\beta 2 M$ dans les urines, chez les travailleurs de plus de 60 ans [20]. Malgré les incertitudes méthodologiques

\footnotetext{
${ }^{5} \mathrm{BMD}_{10}$ : $\mathrm{BMD}$ associée à un niveau de réponse de $10 \%$.

${ }^{6}$ Valeurs supérieures à $285 \mu \mathrm{g} / \mathrm{g}$ de créatinine pour la RBP et à $295 \mu \mathrm{g} / \mathrm{g}$ de créatinine pour la $\beta 2 \mathrm{M}$.
} 
soulevées par Bernard et Lauwerys ${ }^{7}$ [21], cette étude a été retenue pour recommander l'application d'un suivi biologique complémentaire de la RBP et de la $\beta 2 M$ dans les urines lorsque la concentration urinaire de cadmium dépasse $2 \mu \mathrm{g} / \mathrm{g}$ de créatinine. L'objectif d'un tel dispositif est de prendre en compte les paramètres de l'évaluation du risque qui ne peuvent pas être totalement intégrés dans la VLB proposée ; ceux-ci incluent principalement l'âge des travailleurs, la charge rénale de cadmium en raison de sa très longue demi-vie et le tabagisme.

Une VBR pour le cadmium urinaire a également pu être proposée à partir de l'étude française ENNS, en population générale. Les concentrations de cadmium dans les urines, correspondant aux 95èmes pourcentiles des distributions dans cette étude sont de $0,79 \mu \mathrm{g} / \mathrm{g}$ de créatinine chez les non-fumeurs et de $1,00 \mu \mathrm{g} / \mathrm{g}$ de créatinine chez les fumeurs [22]. Les VBR retenues pour le cadmium urinaire sont ainsi de $0,80 \mu \mathrm{g} / \mathrm{g}$ de créatinine chez les non-fumeurs et de $1,00 \mu \mathrm{g} / \mathrm{g}$ de créatinine chez les fumeurs.

Il est à noter que dans le cadre plus global des travaux d'expertise menés, une VLB pragmatique visant à protéger de la toxicité tubulaire et une VBR ont également été recommandées pour le cadmium sanguin. Des VBR de $250 \mu \mathrm{g} / \mathrm{g}$ de créatinine dans les urines pour les indicateurs biologiques d'effets précoces que sont la RBP et la $\beta 2 M$ ont également été préconisés.

\section{VLB pour une substance à seuil d'effet basée sur une exposition à la VLEP-8 $\mathrm{h}$ à partir de corrélations entre les concentrations atmosphériques mesurées et les niveaux d'IBE : cas du styrène [23]}

Pour le styrène, cinq IBE sont disponibles et bien documentés dans la littérature examinée jusqu'en $2011^{8}$.

Deux IBE, le styrène urinaire et la somme des acides mandélique et phénylglyoxylique urinaires, ont été retenus comme pertinents pour la SBEP.

Quatre IBE (styrène dans l'air expiré et dans le sang, les acides mandélique et phénylglyoxilique seuls) ont été exclus en raison des inconvénients liés aux prélèvements (caractère invasif pour le sang), aux modalités de transport et de conservation des échantillons et de difficultés d'ordre pré-analytique.

Les études de terrain disponibles n'ont pas permis d'établir une relation dose-réponse entre les concentrations urinaires de styrène et/ou de la somme des acides mandélique et phénylglyoxylique et des effets sanitaires (altération de l'audition et dyschromatopsie). Il a donc été jugé plus pertinent de prendre en compte les études mettant en relation les concentrations atmosphériques de styrène aux concentrations urinaires des différents IBE retenus pour construire des VLB.

\footnotetext{
7 Les auteurs indiquent que les valeurs de $\beta 2 \mathrm{M}$ urinaire utilisées par Jarup et Elinder (1994) ne sont pas pertinentes pour les personnes de plus de 60 ans (modifications de la physiologie rénale).

8 Styrène dans l'urine, styrène dans le sang, styrène dans l'air expiré, acide mandélique dans l'urine, acide phénylglyoxylique dans l'urine, somme de ces deux acides dans l'urine.
}

Pour le styrène urinaire, trois études de terrain [24-26] ont permis, à partir des équations des droites de régression, de calculer des concentrations urinaires de styrène en fin de poste correspondant à une exposition à la VLEP$8 \mathrm{~h}\left(100 \mathrm{mg} / \mathrm{m}^{3}\right)$. À partir de ces études, la moyenne des concentrations calculées pour une exposition à la VLEP-8 $\mathrm{h}$ est d'environ $40 \mu \mathrm{g} / \mathrm{L}$, valeur retenue comme VLB. De la même manière, 3 études de terrain $[24,26,27]$ ont permis de recommander une VLB de $600 \mathrm{mg} / \mathrm{g}$ de créatinine pour la somme des acides mandélique et phénylglyoxylique urinaires en fin de semaine fin de poste. Il est à noter que, conformément au guide méthodologique, les concentrations urinaires volumiques issues de certaines études ont été rapportées à la créatinine en utilisant la valeur de 1,4 mg de créatinine par litre d'urine.

\section{VLB pour une substance à seuil d'effet basée sur une exposition à la VLEP-8 $\mathrm{h}$ à partir de modèles toxicocinétiques : cas du 2-butoxyéthanol [28]}

La contribution de la pénétration percutanée du 2butoxyéthanol à la charge corporelle et sa toxicité systémique justifient de réaliser une SBEP. Une analyse des données de la littérature jusqu'en 2009 a permis de retenir l'acide 2-butoxyacétique, métabolite toxique du 2-butoxyéthanol, responsable de l'apparition d'un effet systémique, l'hémolyse (études expérimentales in vivo et in vitro). L'acide 2-butoxyacétique peut être dosé dans les urines avant hydrolyse (pour le dosage de l'acide 2butoxyacétique libre) ou après hydrolyse. Étant données les variations intra et interindividuelles des taux de conjugaison de l'acide 2- butoxyacétique dans les urines, le dosage de l'acide 2-butoxyacétique dans l'urine après hydrolyse est jugé plus pertinent que le dosage de l'acide 2-butoxyacétique libre dans les urines.

Une seule étude en milieu professionnel rapporte des données combinées de bio-métrologie (dosage de l'acide 2 butoxyacétique libre urinaire) et d'effets sur la santé [29]. Il s'agit d'expositions professionnelles à des concentrations atmosphériques inférieures à $1 \mathrm{ppm}$. Les concentrations urinaires d'acide 2-butoxyacétique libre en fin de poste sont de l'ordre de $10 \mathrm{mg} / \mathrm{g}$ de créatinine (moyennes) (avec une valeur maximum de $51 \mathrm{mg} / \mathrm{g}$ de créatinine). Les auteurs signalant que ces résultats doivent être confirmés par d'autres études, il n'a pas été jugé possible, à partir des données de la littérature, de tirer des conclusions suffisamment certaines quant à une relation dose-réponse entre les niveaux des IBE et les effets sur la santé.

Bien que la relation entre la concentration interne et les effets toxiques soit à privilégier pour la mise en place d'une valeur pour un IBE, il paraît difficile à partir des études in vitro disponibles [29-32] d'apporter des éléments pertinents concernant une relation éventuelle entre les niveaux des IBE et les effets sur la santé.

Les études rapportant des corrélations entre les concentrations atmosphériques et les niveaux d'IBE présentaient des limites méthodologiques ( 2 études de terrain, 4 études sur volontaires). Elles n'ont pas permis de recommander avec assurance une VLB basée sur une exposition à une VLEP. $8 \mathrm{~h}$ de $49 \mathrm{mg} / \mathrm{m}^{3}$. 
Un modèle PBPK, validé chez l'Homme publié dans la littérature, a permis de déterminer la relation entre l'exposition au 2-butoxyéthanol et des concentrations d'acide 2-butoxyacétique (sang et urine après hydrolyse) [33-35]. Les concentrations urinaires d'acide 2butoxyacétique après hydrolyse obtenues avec ce modèle étaient concordantes avec celles obtenues dans des études sur volontaires [36]. La VLB a été construite sur la base d'une exposition à la VLEP-8h, à partir de ce modèle PBPK en simulant une exposition corps entier (inhalation et cutanée) à $49 \mathrm{mg} / \mathrm{m}^{3}$ pendant 8 heures, au repos. La concentration maximale d'acide 2-butoxyacétique urinaire après hydrolyse prédite à partir de ce modèle est de $100 \mathrm{mg} / \mathrm{g}$ de créatinine. Une VLB de $100 \mathrm{mg} / \mathrm{g}$ de créatinine pour l'acide 2-butoxyacétique urinaire a ainsi été recommandée en se basant sur une exposition au 2-butoxyéthanol correspondant à la VLEP-8 $\mathrm{h}$ de $49 \mathrm{mg} / \mathrm{m}^{3}$ [28].

\section{VLB/VBR pour une substance sans seuil d'effet : cas de l'acrylamide [37]}

En l'absence de mécanisme d'action clairement élucidé, l'acrylamide a été considéré par défaut comme un cancérogène sans seuil. Ainsi en 2011, les experts de l'Anses avaient émis des recommandations d'ERI de cancer (mésothéliome testiculaire) calculés à partir de données chez le rat [38]. Parmi les 8 IBE identifiés dans la littérature jusqu'en 2011, seuls les adduits N-(2-carbamoyléthyl)valine (AAVal) de l'acrylamide à l'hémoglobine, mesurés dans le sang, ont été retenus comme IBE pertinents pour la SBEP. Les moments de prélèvements sont indépendants du moment de la journée ou de la semaine car les adduits à l'hémoglobine sont stables (pendant la durée de vie des érythrocytes soit 120 jours) sous réserve que les prélèvements soient réalisés après un minimum de 120 jours d'exposition.

Concernant l'élaboration de la VLB, aucune donnée chez l'homme n'a permis de relier des concentrations d'adduits à l'hémoglobine (AAval) aux données de cancérogénicité de l'acrylamide.

La construction de VLB basées sur différentes concentrations atmosphériques a donc été envisagée à partir d'études de terrain ou d'études expérimentales.

\section{VLB à partir d'études de terrain}

Les études en milieu professionnel procurant des niveaux d'exposition atmosphérique à l'acrylamide sont rares. Les études d'exposition à l'acrylamide concernent davantage la population générale et permettent d'estimer les doses d'acrylamide ingérées à partir des concentrations d'adduits d'acrylamide à l'hémoglobine. Jones et al. ont étudié l'association entre les concentrations atmosphériques d'acrylamide et les taux d'adduits (AAVal) chez 60 travailleurs au Royaume-Uni [39]. Les concentrations atmosphériques d'acrylamide ne sont pas rapportées, seule la moyenne d'environ $30 \mu \mathrm{g} / \mathrm{m}^{3}$ est indiquée. Les auteurs indiquent qu'il existe une forte corrélation $(r=0,61)$ entre les concentrations atmosphériques d'acrylamide et les concentrations sanguines d'AAVal. Cependant, dans cette étude, la limite de détection (LD) du dosage de l'acrylamide dans l'air est égale à $5 \mu \mathrm{g} / \mathrm{m}^{3}$. Elle est donc supérieure à la concentration atmosphérique d'acrylamide de
$4 \mu \mathrm{g} / \mathrm{m}^{3}$ estimée en 2011 comme correspondant à l'ERI de cancer de $10^{-4}$ pour 40 ans d'exposition [38]. De plus, la corrélation rapportée dans cette publication a été calculée pour des expositions allant de 5 à $200 \mu \mathrm{g} / \mathrm{m}^{3}$ (déterminées graphiquement), concentrations supérieures aux concentrations atmosphériques d'acrylamide calculées également pour des ERI de $10^{-5}\left(0,4 \mu \mathrm{g} / \mathrm{m}^{3}\right)$ et $10^{-6}\left(0,04 \mu \mathrm{g} / \mathrm{m}^{3}\right)$. Il n'est donc pas possible de connaître la validité de la corrélation rapportée pour une exposition aux concentrations atmosphériques inférieures à la LD de cette étude.

\section{VLB à partir de données expérimentales}

Des concentrations ont pu être calculées pour 3 ERI pour le mésothéliome testiculaire à partir des données chez le rat. Toutefois, elles introduisent de nombreuses incertitudes. En effet, les paramètres cinétiques de l'acrylamide et des adduits ont été mesurés pour une absorption par voie orale d'une dose unique d'acrylamide. En outre, la constante de formation des adduits n'a pas été déterminée pour une exposition par inhalation ni pour une exposition continue avec une concentration d'adduits à l'équilibre.

En conclusion, les calculs des concentrations d'AAVal en fonction, soit de l'étude chez le rat retenue pour construire la VLEP, soit des concentrations atmosphériques d'acrylamide issues des études de terrain disponibles présentent de nombreuses incertitudes. Ces deux approches ne sont pas suffisamment robustes pour prédire avec assurance des concentrations d'adduits de l'acrylamide à l'hémoglobine (AAval) associées à trois ERI $\left(10^{-4}, 10^{-5}\right.$ et $\left.10^{-6}\right)$.

Les autres effets de l'acrylamide rapportés en milieu professionnel concernent le plus souvent la neurotoxicité. Seules deux études mettent en relation des effets neurotoxiques (examen clinique, index de neurotoxicité) et des concentrations sanguines des adduits de l'acrylamide à l'hémoglobine (AAVal) [40-42]. Ces deux études présentent certaines limites :

- dans l'étude de Calleman et al., quels que soient les symptômes de neuropathie étudiés, les No-Observed-Adverse Effect Level (NOAEL) sont égaux à 2000 pmol/g de globine d'AAVal et les Lowest-Adverse Effect Level (LOAEL) sont égaux à $6000 \mathrm{pmol} / \mathrm{g}$ de globine d'AAVal [40]. Cette relation dose-réponse étant la même quelle que soit la précocité des effets, cela limite l'interprétation de ces doses repères pour établir une relation dose-réponse ;

- dans l'étude de Hagmar et al., le décalage temporel entre la fin des expositions, la mesure des concentrations d'adduits et l'étude des effets sanitaires rend l'interprétation et l'extrapolation délicates [42].

Il n'a donc pas été jugé pertinent de recommander de VLB sur la base d'un autre effet que le cancer.

\section{VBR}

Seules des VBR ont ainsi été proposées pour les adduits de l'acrylamide dans le sang à l'hémoglobine AAVal. À défaut de données françaises en population générale, l'étude de Vesper et al. portant sur une population européenne a été retenue [43]. Cette étude rapporte des concentrations d'adduits de l'acrylamide chez 510 sujets (entre 40 et 60 ans) en fonction du statut tabagique. Ainsi, les VBR 
recommandées à partir du $95^{\mathrm{e}}$ pourcentile de la distribution des valeurs retrouvé dans cette enquête en fonction du statut tabagique, sont pour les adduits de l'acrylamide à l'hémoglobine AAVal dans le sang : $85 \mathrm{pmol} / \mathrm{g}$ de globine (non-fumeurs) et $285 \mathrm{pmol} / \mathrm{g}$ de globine (fumeurs).

Il est à noter qu'une alimentation riche en chips, frites, céréales, café de même que le tabagisme peuvent apporter une importante contribution à la concentration d'AAVal au sein de la population générale. Enfin les médicaments inducteurs ou inhibiteurs de CYP2E1 peuvent également influencer la formation des adduits AAVal dans le sang.

\section{Conclusion}

Les recommandations de valeurs biologiques françaises se font en suivant une méthodologie structurée et rendue publique. À ce jour, outre les substances évoquées dans cet article, des recommandations sur le toluène, le di(2-éthylhexyl)phtalate, le di-n-butylphtalate, le butylbenzyl-phtalate et le chrome hexavalent sont disponibles sur le site Internet de l'Anses (cf lien : https: / / www.anses. fr/fr/content/avis-du-ces-expertise-envue-de-la-fixation-de-valeurs-limites-dexposition-\%C3 \%A0des-agents). Des recommandations de valeurs biologiques pour le cobalt, le béryllium, le 1,3-butadiène, le plomb, le perchloroéthylène, le dichlorométhane devraient également faire l'objet de publications dans le futur.

\section{Déclaration de liens d'intérêts}

Les auteurs déclarent ne pas avoir de liens d'intérêts.

\section{Références}

[1] Pillière F. Erratum à «Introduction : définition, contexte réglementaire, valeurs de référence et états des lieux de la surveillance biologique des expositions » [Arch Mal Prof Environ 2008 ; 69(2) : 306]. Arch Mal Prof Environ 2008;69:636.

[2] Anses, $122 \mathrm{p}$ Valeurs limites d'exposition en milieu professionnel - document de référence pour la construction et la mesure de valeurs limites d'exposition à des agents chimiques en milieu professionnel. Anses: Maisons-Alfort; 2014.

[3] Société française de médecine du travail. Surveillance biologique des expositions professionnelles aux agents chimiques Recommandations de bonne pratique TM37. Références en santé travail; 2016. p. 29.

[4] Cocker J. A perspective on biological monitoring guidance values. Toxicol Lett 2014;231:122-5.

[5] Pillière F, Cointot MLS. Biological reference values for occupational exposures. Arch Mal Prof Environ 2012;73:451-4.

[6] Cocker J, Jones K, Morton J, Mason HJ. Biomonitoring at the UK health and safety laboratory. Int J Hyg Environ Health 2007;210:383-6.

[7] Drexler H, Göen T, Schaller KH. Biological tolerance values: change in a paradigm concept from assessment of a single value to use of an average. Int Arch Occup Environ Health 2008;82:139-42.

[8] Göen T, Schaller KH, Drexler H. Biological reference values for chemical compounds in the work area (BARs): an approach for evaluating biomonitoring data. Int Arch Occup Environ Health 2012;85:571-8.
[9] Morgan MS. The biological exposure indices: a key component in protecting workers from toxic chemicals. Environ Health Perspect 1997;105:105-15.

[10] SCOEL. Methodology for the derivation of occupational exposure limits: key documentation version 7. Luxembourg: European Commission; 2013 [38 p].

[11] Bonvallot N, Dor F. Valeurs toxicologiques de référence : méthodes d'élaboration; 2002 [Institut de Veille Sanitaire; $84 \mathrm{p}]$.

[12] Anses. Valeurs limites d'exposition en milieu professionnel - évaluation des indicateurs biologiques d'exposition et recommandation de valeurs biologiques de référence pour le cadmium et ses composés - rapport d'expertise collective ; à paraître, document soumis à consultation. $68 \mathrm{p}$.

[13] SCOEL. Recommendation from the Scientific Committee on occupational exposure limits for cadmium and its inorganic compounds - SCOEL/SUM/138. Luxembourg: European Commission; 2010 [26 p].

[14] Bernard A. Cadmium \& its adverse effects on human health. Indian J Med Res 2008;128:557-64.

[15] Hotz P, Buchet JP, Bernard A, Lison D, Lauwerys R. Renal effects of low-level environmental cadmium exposure: 5-year follow-up of a subcohort from the Cadmibel study. Lancet 1999;354:1508-13.

[16] Jarup L, Berglund M, Elinder CG, Nordberg G, Vahter M. Erratum: health effects of cadmium exposure - a review of the literature and a risk estimate (Scandinavian Journal of Work, Environment and Health (1998) 24, suppl 1 (52)). Scand J Work Environ Health 1998;24:240.

[17] Prozialeck WC, Edwards JR. Early biomarkers of cadmium exposure and nephrotoxicity. BioMetals 2010;23:793-809.

[18] Chaumont A, De Winter F, Dumont X, Haufroid V, Bernard $A$. The threshold level of urinary cadmium associated with increased urinary excretion of retinol-binding protein and $\beta 2$-microglobulin: a re-assessment in a large cohort of nickel-cadmium battery workers. Occup Environ Med 2011;68:257-64.

[19] EFSA. Technical report of EFSA prepared by assessment methodology unit on meta-analysis of dose-effect relationship of cadmium for benchmark dose evaluation. EFSA Sci Rep 2009:1-62.

[20] Järup L, Elinder CG. Dose-response relations between urinary cadmium and tubular proteinuria in cadmium-exposed workers. Am J Ind Med 1994;26:759-69.

[21] Bernard AM, Lauwerys RR, Jarup L, Elinder CG. Dose-response relations between urinary cadmium and tubular proteinuria in adult workers. Am J Ind Med 1997;31:116-20.

[22] Fréry N, Saoudi A, Garnier R, Zeghnoun A, Falq G. Exposition de la population française aux substances chimiques de l'environnement. Saint-Maurice: Institut de veille sanitaire; 2011. p. 58.

[23] Anses. Valeurs limites d'exposition en milieu professionnel évaluation des indicateurs biologiques d'exposition pour le styrène en vue de la construction de valeurs limites biologiques avis de l'Anses - rapport d'expertise collective. Anses: MaisonsAlfort; 2014 [68 p].

[24] Gobba F, Galassi C, Ghittori S, Imbriani M, Pugliese F, Cavalleri A. Urinary styrene in the biological monitoring of styrene exposure. Scand J Work Environ Health 1993;19:175-82.

[25] Maestri L, Mestad IJ, Imbriani M. Mercapturates and biologic monitoring: styrene. G Ital Med Lav Ergon 1999;21: 334-40.

[26] Ong CN, Shi CY, Chia SE, Chua SC, Ong HY, Lee BL, et al. Biological monitoring of exposure to low concentrations of styrene. Am J Ind Med 1994;25:719-30.

[27] De Rosa E, Bartolucci GB, Perbellinie L, Brugnone F, Rausa G. Environmental and biological monitoring of exposure to toluene, styrene, and n-hexane. Appl Ind Hyg 1988;3:332-7. 
[28] Anses. Valeurs limites d'exposition en milieu professionnel indicateurs et valeurs biologiques pour le 2-butoxyéthanol et son acétate - avis de l'Anses - rapport d'expertise collective. Anses: Maisons-Alfort; 2010 [46 p].

[29] Haufroid V, Thirion F, Mertens P, Buchet JP, Lison D. Biological monitoring of workers exposed to low levels of 2 butoxyethanol. Int Arch Occup Environ Health 1997;70:232-6.

[30] Udden MM. Hemolysis and deformability of erythrocytes exposed to butoxyacetic acid, a metabolite of 2-butoxyethanol: II. Resistance in red blood cells from humans with potential susceptibility. J Appl Toxicol 1994;14:97-102.

[31] Udden MM. Rat erythrocyte morphological changes after gavage dosing with 2-butoxyethanol: a comparison with the in vitro effects of butoxyacetic acid on rat and human erythrocytes. J Appl Toxicol 2000;20:381-7.

[32] Udden MM. In vitro sub-hemolytic effects of butoxyacetic acid on human and rat erythrocytes. Toxicol Sci 2002;69:258-64.

[33] Corley RA, Bormett GA, Ghanayem BI. Physiologically based pharmacokinetics of 2-butoxyethanol and its major metabolite, 2-butoxyacetic acid, in rats and humans. Toxicol Appl Pharmacol 1994;129:61-79.

[34] Corley RA, Markham DA, Banks C, Delorme P, Masterman A, Houle JM. Physiologically based pharmacokinetics and the dermal absorption of 2-butoxyethanol vapor by humans. Fundam Appl Toxicol 1997;39:120-30.

[35] Franks SJ, Spendiff MK, Cocker J, Loizou GD. Physiologically based pharmacokinetic modelling of human exposure to 2butoxyethanol. Toxicol Lett 2006;162:164-73 [2-3 SPEC. ISS.].

[36] Jones K, Cocker J. A human exposure study to investigate biological monitoring methods for 2-butoxyethanol. Biomarkers 2003;8:360-70.
[37] Anses. Valeurs limites d'exposition en milieu professionnel - évaluation des indicateurs biologiques d'exposition pour l'acrylamide en vue de la construction de valeurs limites biologiques - avis de l'Anses - rapport d'expertise collective. Anses: Maisons-Alfort; 2017 [76 p].

[38] Anses. Valeurs limites d'exposition en milieu professionnel évaluation des effets sur la santé et des méthodes de mesure des niveaux d'exposition sur le lieu de travail pour l'acrylamide [No CAS : 79-06-1] Avis de l'Anses - rapport d'expertise collective. Anses: Maisons-Alfort; 2011.

[39] Jones K, Garfitt S, Emms V, Warren N, Cocker J, Farmer P. Correlation of haemoglobin-acrylamide adducts with airborne exposure: an occupational survey. Toxicol Lett 2006;162:174-80 [2-3 SPEC. ISS.].

[40] Calleman CJ. The metabolism and pharmacokinetics of acrylamide: implications for mechanisms of toxicity and human risk estimation. Drug Metabol Rev 1996;28:527-90.

[41] Calleman CJ, Wu Y, He F, Tian G, Bergmark E, Zhang S, et al. Relationships between biomarkers of exposure and neurological effects in a group of workers exposed to acrylamide. Toxicol Appl Pharmacol 1994;126:361-71.

[42] Hagmar L, Törnqvist M, Nordander C, Rosén I, Bruze M, Kautiainen $A$, et al. Health effects of occupational exposure to acrylamide using hemoglobin adducts as biomarkers of internal dose. Scand J Work Environ Health 2001;27:219-26.

[43] Vesper HW, Slimani N, Hallmans G, Tjønneland A, Agudo A, Benetou V, et al. Cross-sectional study on acrylamide hemoglobin adducts in subpopulations from the European Prospective Investigation into Cancer and Nutrition (EPIC) study. J Agric Food Chem 2008;56:6046-53. 